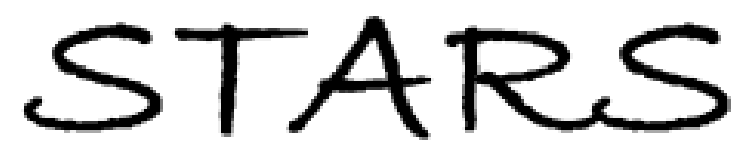

University of Central Florida

STARS

$1-1-2010$

\title{
First-principles study of formation of Se submonolayer structures on Ru surfaces
}

Sergey Stolbov

University of Central Florida

Find similar works at: https://stars.library.ucf.edu/facultybib2010 University of Central Florida Libraries http://library.ucf.edu

This Article is brought to you for free and open access by the Faculty Bibliography at STARS. It has been accepted for inclusion in Faculty Bibliography 2010 s by an authorized administrator of STARS. For more information, please contact STARS@ucf.edu.

\section{Recommended Citation}

Stolbov, Sergey, "First-principles study of formation of Se submonolayer structures on Ru surfaces" (2010). Faculty Bibliography 2010s. 829.

https://stars.library.ucf.edu/facultybib2010/829

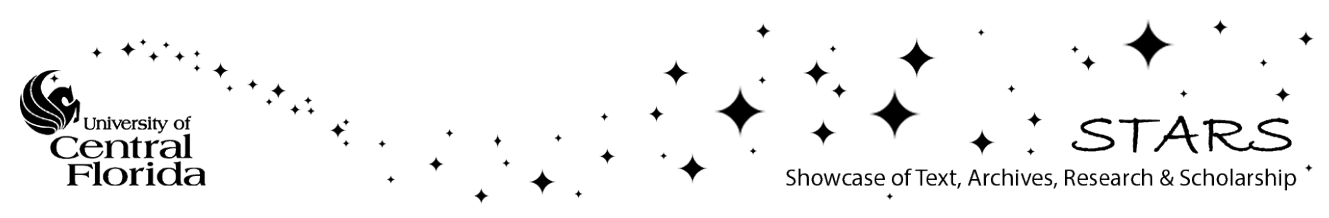




\title{
First-principles study of formation of Se submonolayer structures on Ru surfaces
}

\author{
Sergey Stolbov \\ Department of Physics, University of Central Florida, Orlando, Florida 32816, USA \\ (Received 30 June 2010; revised manuscript received 27 August 2010; published 29 October 2010)
}

\begin{abstract}
The $\mathrm{Ru}$ nanoparticles with Se submonolayer coverage $(\mathrm{Se} / \mathrm{Ru})$ demonstrate high electrocatalytic activity toward oxygen reduction reaction (ORR) on cathodes of proton exchange membrane fuel cells. To understand the mechanisms of formation of Se structures on Ru surfaces, the geometric and electronic structures and energetics have been calculated in the present work for various distributions of Se atoms on the $\mathrm{Ru}(0001)$ surface and in the vicinity of the edge between the (0001) and (1101) facets. The calculations were performed within the density-functional theory with plane-wave expansion for wave functions and the projector augmented wave potentials. It has been found that due to electronic charge transfer from $\mathrm{Ru}$ to Se upon selenium adsorption, Se atoms become negatively charged and repel each other. This repulsion makes compact Se islands on $\mathrm{Ru}(0001)$ unstable. Se atoms prefer to separate from each other by the distance of $\sim 5.47 \AA$ or larger, which is possible for all Se adsorbates if coverage is not exceeding 1/3 ML. Further increase in Se coverage weakens Se-Ru bonding. Three-dimensional Se structure such as 4- and 11-atom pyramids are found to decompose spontaneously with scattering of Se atoms over the $\mathrm{Ru}(0001)$ surface. The Se adsorbates are also found to repel in the vicinity of the edge between the $\mathrm{Ru}$ facets, and a small increase in Se bonding to undercoordinated $\mathrm{Ru}$ atom does not change the trend of Se adsorbates to separate from each other. The obtained most stable configurations of Se on Ru with 1/3 ML coverage or less may also be optimal for ORR because they provide $\mathrm{Ru}$ sites available for $\mathrm{O}$ and $\mathrm{OH}$ adsorption.
\end{abstract}

DOI: $10.1103 /$ PhysRevB.82.155463

PACS number(s): 68.43.Fg, 71.15.Mb, 68.43.Hn, 73.20.-r

\section{INTRODUCTION}

Proton exchange membrane fuel cell (PEMFC) and direct methanol fuel cells (DMFCs) are clean sources of electric power with low operating temperature which are considered key elements of emerging hydrogen economy. However, the cost of these fuel cells is unacceptably high. Since the Ptbased catalysts used in both electrodes are the most expensive parts of these fuel cells, search for new cost-effective electrocatalytic materials is critical for their practical application. Furthermore, performance of both PEMFC and DMFC suffers from a low rate of electrocatalytic oxygen reduction reaction $(\mathrm{ORR})$ on the $\mathrm{Pt}$ cathode, which results in a reduced onset potential and, therefore low fuel cell efficiency. ${ }^{1}$ It is thus not surprising that so much effort has been made in searching for new materials with improved electrocatalytic properties.

Based on experimental evidence, one of the directions of this search is exploring Ru-based materials as potential cathodes for the fuel cells. The earliest significant finding in this area was reported more than two decades ago, when AlonsoVante and Tributsch found the Chevrel phase-type compound $\mathrm{Mo}_{4.2} \mathrm{Ru}_{1.8} \mathrm{Se}_{8}$ to have a high ORR rate. ${ }^{2}$ It has later been shown, however, that the best electrocatalytic performance is achieved with Ru-Se-based structures other than Chavrel phases. ${ }^{3-11}$ Recent studies show that most of these materials form nanoparticles with composition described by formula $\mathrm{Ru}_{x} \mathrm{Se}_{y}$, while their geometric structure is determined by particular condition of synthesis. These electrocatalysts demonstrate high, comparable to $\mathrm{Pt}$, rate of ORR, as well as selectivity to water (i.e., a small amount of $\mathrm{H}_{2} \mathrm{O}_{2}$ production), and high stability toward oxidation and methanol tolerance. These properties are found, however, to depend strongly on the composition and geometric structure of the system. The pathways and rate of ORR are essentially determined by energetics of adsorption of reactants and intermediates, in particular, the adsorption of $\mathrm{OH}$ and atomic oxygen, ${ }^{12,13}$ which in turn depend on the electronic and geometric structures of the catalyst surface.

The mechanisms underlying electrocatalytic activity of a material can thus be understood only if the geometric structure of its surface is known. This is why much effort has been made to obtain the composition and structural characteristics of new $\mathrm{Ru}_{x} \mathrm{Se}_{y}$ systems with enhanced activity toward ORR. By changing treatment conditions, Shen et al. ${ }^{8}$ have obtained this type of $\mathrm{Ru}_{x} \mathrm{Se}_{y}$ nanostructures with $\mathrm{Ru}$ hcp core and Se shell, and other ones apparently with pyrite structure, or mixture of the two. Vogel et al. ${ }^{3}$ by treatment of $\mathrm{Ru}_{4} \mathrm{Se}_{2}(\mathrm{CO})$ at various temperatures in $\mathrm{He}$ and $\mathrm{N}_{2}$ atmosphere, have synthesized nanoparticles with dominating content of $\mathrm{Ru}$ in the form of hcp clusters and Se somehow distributed on the cluster surfaces, as well as small amount of $\mathrm{RuSe}_{2}$ particles with the pyrite structure. There was no direct evidence that $\mathrm{Se}$ was located on the Ru facets but the authors provided implicit arguments in favor of such geometry. Their density-functional theory (DFT)-based $a b$ initio thermodynamics analysis suggests that even under the condition of thermodynamically stable bulk $\mathrm{RuSe}_{2}$ pyrite structure, Serich surface is preferred which suggests Ru-Se segregation. Similar structures have been obtained by Liu et al.,$^{5}$ who synthesized $\mathrm{Ru}_{85} \mathrm{Se}_{15}$ nanoparticles, for which $\mathrm{x}$-ray diffraction also showed significant $\mathrm{Ru}$ hcp content suggesting the Ru-core-Se-shell geometry. Delacôte et al., ${ }^{4}$ using an aqueous-based synthesis method, have also obtained $\mathrm{Ru}$ nanoparticles with hcp structure presumably surrounded by Se atoms. Detailed analysis of the results of the anomalous small-angle $\mathrm{x}$-ray scattering experiments performed by Zehl et $a l .{ }^{6}$ brought them to the conclusion that synthesized carbon-supported $\mathrm{Ru}-\mathrm{Se}$ catalysts form $\mathrm{Ru}$ hcp clusters with 


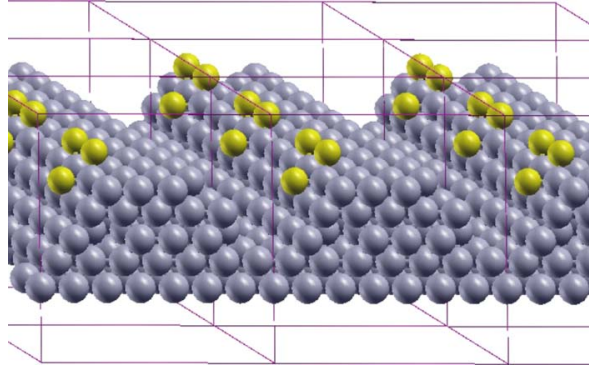

FIG. 1. (Color online) A periodic structure modeling the edge between $\mathrm{Ru}(0001)$ and $\mathrm{Ru}(1101)$ facets with three Se atoms (yellow balls) adsorbed in the vicinity of the edge. Gray balls represent $\mathrm{Ru}$ atoms.

average size of $2.2 \mathrm{~nm}$ decorated with small Se islands. Zaikovskii et al. ${ }^{7}$ have obtained similar structures, however, they suggest that the $\mathrm{Ru}$ particles are covered with $\mathrm{Ru}$ selenide clusters of $<1 \mathrm{~nm}$ size whose structures are not well determined but most likely do not have the $\mathrm{RuSe}_{2}$ pyrite structure.

The above results thus suggest that the ORR active $\mathrm{Ru}-\mathrm{Se}$ nanoparticles have sizes in the range between 1 and $6 \mathrm{~nm}$ while in most cases it is $\sim 2-3 \mathrm{~nm} .{ }^{3-11}$ These particles have a Ru metal core with bulklike hcp structure and Se shell with submonolayer coverage. However, it is not clear whether Se atoms scatter over the particle surface or form Se or even $\mathrm{Se}_{x} \mathrm{Ru}_{y}$ islands. The goal of the present work is to find equilibrium configurations of these structures using the firstprinciples computational approach based on DFT.

\section{COMPUTATIONAL DETAILS}

Since the 2-4 $\mathrm{nm}$ size particles observed in experimental works are too large for direct first-principles calculations, I use two models to approximate the Ru-core clusters. The flat facets of the particles are approximated by a $\mathrm{Ru}(0001)$ surface (the most stable surface of $\mathrm{Ru}$ ) while the regions in the vicinity of their edges between (0001) and (1101) facets are modeled by the periodic structure with large translation vectors shown in Fig. 1. Our recent studies of similar systems ${ }^{14,15}$ confirm this approximation to provide a sufficient accuracy.

For all systems under consideration, the energetics and equilibrium atomic configurations are obtained using the VASP5. 2 code $^{16}$ with projector augmented wave potentials ${ }^{17}$ and the Perdew-Burke-Ernzerhof version of the generalized gradient approximation for the exchange and correlation functional. ${ }^{18}$ In order to maintain periodicity for Se submonolayer structures on $\mathrm{Ru}(0001)$ I use supercells with a five-layer $\mathrm{Ru}(0001)$ slab and vacuum layer of $15 \AA$. For the Se structures containing one or two adsorbate atoms, a (3 $\times 3)$ in-plane unit was used while for those with larger number of Se atoms I used a $(4 \times 4)$ supercell. The $(4 \times 4 \times 1)$ and $(3 \times 3 \times 1) k$-point samplings in Brillouin zone were used for the smaller and larger supercells, respectively.

In order to model the effects of edge between $\mathrm{Ru}(0001)$ and (1101) facets in the particle, I used a supercell with 7 $\times 4$ in-plane periodicity with a 116-Ru atom slab constructed by stacking five $\mathrm{Ru}(0001)$ layers: two of $7 \times 4$, one of 6 $\times 4$, one of $5 \times 4$, and one of $4 \times 4$ atoms (see Fig. 1). This structure thus forms a four-atom wide $\mathrm{Ru}(0001)$ facet and two $\mathrm{Ru}(1101)$ facets The bottom two layers are not allowed to relax to guarantee the stability of the superstructure. The supercell also included a $15 \AA$ vacuum layer separating the slabs along the direction perpendicular to the (0001) facet. The Brillouin zone is sampled with a $(2 \times 3 \times 1) k$-point mesh.

To characterize the strength of bonding of a single Se atom to a $\mathrm{Ru}$ surface or nanostructure I use the binding energy,

$$
E_{b}(\mathrm{Se})=E_{\text {tot }}(\mathrm{Ru} \mathrm{slab})+E_{\text {tot }}(\mathrm{Se} \text { atom })-E_{\text {tot }}(\mathrm{Se} / \mathrm{Ru} \text { slab }),
$$

where the three $E_{\text {tot }}$ terms denote the total energies per supercell calculated for the clean $\mathrm{Ru}$ slab, isolated $\mathrm{Se}$ atom, and the slab with Se adsorbed on the surface, respectively. Given the total energies of stable systems are negative, $E_{b}(\mathrm{Se})$ is positive if the adsorption of atomic Se on the surface is favorable. For multiple adsorbate systems, especially islands, the formation energy is more meaningful,

$$
\begin{aligned}
E_{\text {form }}(\mathrm{Se})= & E_{\text {tot }}(\mathrm{Ru} \text { slab })+n E_{\text {tot }}(\mathrm{Se} \text { atom }) \\
& -E_{\text {tot }}(n \mathrm{Se} / \mathrm{Ru} \mathrm{slab}),
\end{aligned}
$$

where $n$ is the number of Se atoms per supercell on the surface. The formation energy per adsorbate atom $E_{\text {form }} / n$ is more suitable to characterize the average binding energy of the adsorbate in the system.

\section{RESULTS AND DISCUSSION}

In this work, the optimized geometric structures and energetic of the $\mathrm{Se} / \mathrm{Ru}$ system have been calculated for the following initial configurations: (a) various two-dimensional distributions of one, two, three, four, and seven Se atoms on $\mathrm{Ru}(0001)$, including Se islands; (b) 4- and 11-Se atom pyramids; (c) surface Ru atom-Se atom exchange; (d) 1 and 2 ML of Se on $\mathrm{Ru}(0001)$; (f) Ru/Se double layer on $\mathrm{Ru}(0001)$; (g) one, two, and three Se atoms adsorbed on various sites in the vicinity of the edge between (0001) and (1101) facets.

First, adsorption of a $\mathrm{Se}$ atom on $(3 \times 3) \mathrm{Ru}(0001)$ has been calculated. This corresponds to $1 / 9 \mathrm{ML}$ Se coverage, for which the Se-Se interaction is negligible and the obtained characteristics with sufficient accuracy correspond to the single atom adsorption. For Se on hep and fcc hollow sites, the calculated $E_{b}$ is found to be $5.26 \mathrm{eV}$ and $5.15 \mathrm{eV}$, respectively, which makes the hcp sites of $\mathrm{Ru}(0001)$ to be preferred for Se atom adsorption.

Next, the energetics and geometric structure of Se islands on $\mathrm{Ru}$ have been studied. The islands were formed by placing Se atoms on neighboring hcp hollow sites of $\mathrm{Ru}(0001)$ with an initial Se-Se bond lengths of $2.733 \AA$. In the course of relaxation, however, $\mathrm{Se}-\mathrm{Se}$ distances increased dramatically for all considered island. For example, in the relaxed systems they were found to be $3.278 \AA$ for a Se dimer and $3.447 \AA$ for a Se tetramer. This increase for the Se tetramer by $0.714 \AA$ is thus larger than that $(0.545 \AA)$ for the smaller Se dimer. Interestingly, for the seven-atom island (see Fig. 2) 


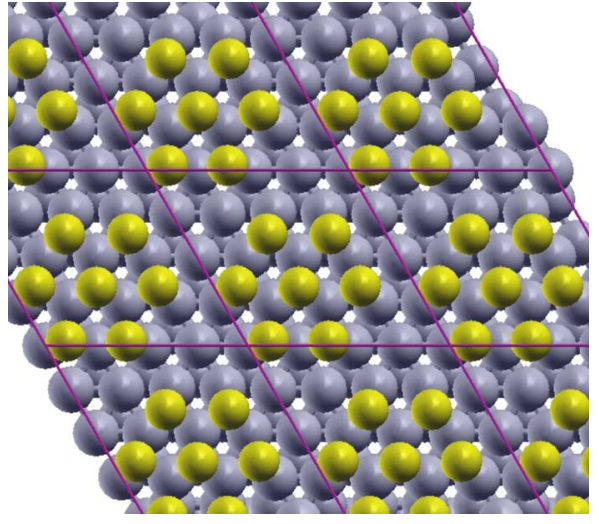

FIG. 2. (Color online) Seven-Se atom island on $\mathrm{Ru}(0001)$ (optimized geometry).

the increase is $0.606 \AA$ between the central and edge Se atoms and $0.687 \AA$ A between two neighboring Se edge atoms. The fact that these numbers are smaller than that for the tetramer can be explained as a result of a noticeable interaction between neighboring islands in the periodic (4 $\times 4) \mathrm{Ru}(0001)$ structure. The energetics of the Se island on $\mathrm{Ru}(0001)$ has been also calculated. The formation energies per atom $\left(E_{\text {form }} / n\right)$, where $n$ is the number of Se atom in the island, are provided for the two-, three-, four-, and sevenatom islands in the second row of Table I. One can see that $E_{\text {form }} / n$ decreases with increasing size of the island. From these results (both energetics and trends in structural relaxation) I can conclude that $\mathrm{Se}$ atoms adsorbed on $\mathrm{Ru}(0001)$ repel each other.

To understand the nature of this repulsion and the character of chemical binding in the system, let us analyze its electronic structure. Shown in Fig. 3, are local densities of electronic states of $\mathrm{Se}$ adsorbates and $\mathrm{Ru}$ surface atoms calculated for the $(3 \times 3)$ unit cell. One can see that the $d \mathrm{Ru}$ states do not change significantly upon Se adsorption. The lowest energy peak and high energy tail in $p$ Se states result from hybridization with the $d \mathrm{Ru}$ states. Since only minor part of the $p$ Se states is involved in this hybridization and only small amount of these states are nonoccupied, one may conclude that the Se-Ru covalent binding is weak in the system. To evaluate the ionic contribution to the binding, the valence charge-density redistribution upon Se adsorption on the Ru surface was analyzed. This characteristic is defined as follow:

$$
\delta \rho(\mathbf{r})=\rho_{\mathrm{SeRu}}(\mathbf{r})-\rho_{\mathrm{Ru}}(\mathbf{r})-\rho_{\text {Se atom }}(\mathbf{r}),
$$

where the right-side equation terms denote the valence charge densities of the $\mathrm{Ru}$ surface adsorbed with Se, clean

TABLE I. Formation energies per atom calculated for Se structures on $\mathrm{Ru}(0001)$.

\begin{tabular}{lcccc}
\hline Number of Se atoms per supercell & 2 & 3 & 4 & 7 \\
\hline$E_{\text {form }} / n(\mathrm{eV})$, Se islands & 5.06 & 5.01 & 4.85 & 4.56 \\
$E_{\text {form }} / n(\mathrm{eV})$, decomposed structures & 5.20 & 5.21 & 5.24 & \\
\hline \hline
\end{tabular}

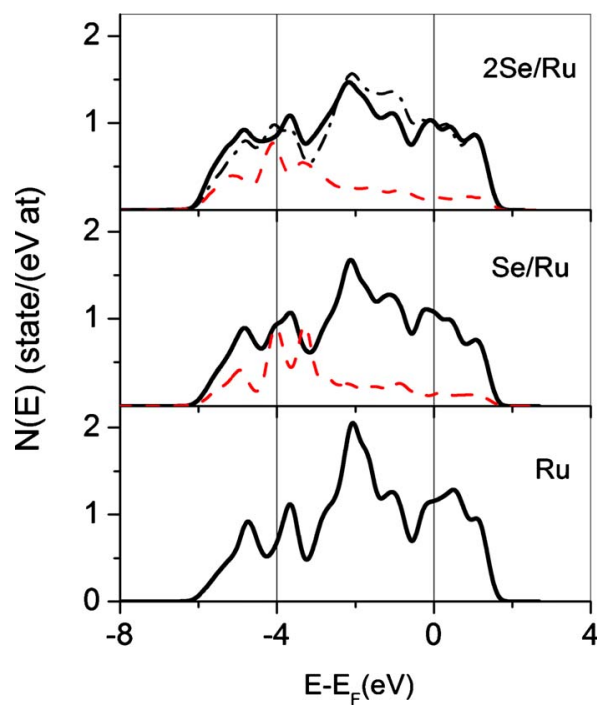

FIG. 3. (Color online) Local densities of electronic states calculated for the clean $\mathrm{Ru}(0001), \mathrm{Se} /(3 \times 3) \mathrm{Ru}(0001)$, and $2 \mathrm{Se} /(3$ $\times 3) \mathrm{Ru}(0001)$. The dashed and solid lines represent the $p$ states of Se and $d$ states of Ru neighboring to the Se-Se dimer, respectively, while the dashed-dotted line corresponds to the $d$ states of Ru surface atom located between two Se atoms.

$\mathrm{Ru}$ surface, and isolated Se atom, respectively. To make this difference tractable, $\rho_{\mathrm{Ru}}(\mathbf{r})$ was calculated for the clean surface with positions of $\mathrm{Ru}$ relaxed on Se adsorption and $\rho_{\text {Se atom }}(\mathbf{r})$ were centered at positions of Se adsorbates. The $\delta \rho(\mathbf{r})$ was calculated for $\mathrm{Ru}(0001)$ with two Se atoms adsorbed on neighboring hcp sites. The plot in Fig. 4 represents a cut of $\delta \rho(\mathbf{r})$ by a plane that is perpendicular to the surface and includes the centers of both Se atoms. The wide black area above the $\mathrm{Ru}$ surface atoms and large white "clouds" around Se reflect a significant electronic charge transfer from the surface to the Se adsorbates typical for strong ionic binding. It is clearly seen from the plot that the accumulated electronic charge density is distributed not symmetrically

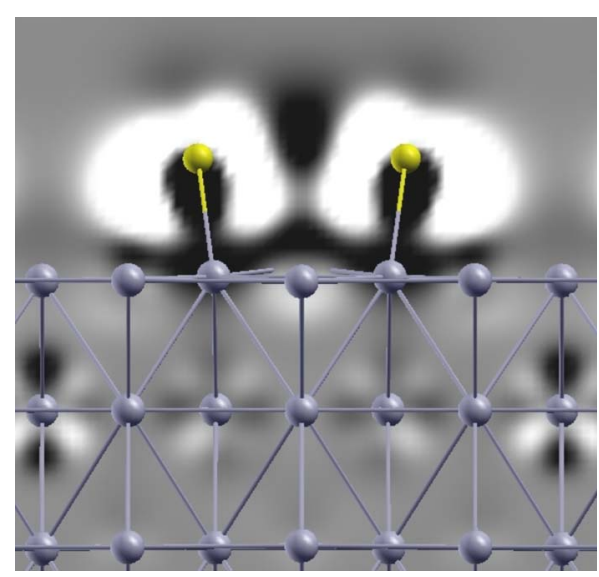

FIG. 4. (Color online) A cut of the electronic charge-density redistribution upon $\mathrm{Se}$ adsorption calculated for $2 \mathrm{Se} /(3$ $\times 3) \mathrm{Ru}(0001)$ using Eq. (3). Black, gray, and white areas correspond to the negative, zero, and positive values of $\delta \rho(\mathbf{r})$, respectively. 


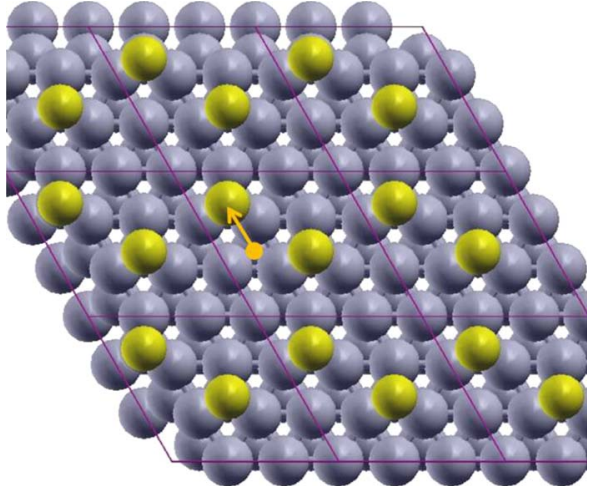

FIG. 5. (Color online) The structure obtained by decomposition of $\mathrm{Se}$ dimer on $\mathrm{Ru}(0001)$. The arrow connects the initial and final states of the decomposition.

with respect to Se atom centers. These electronic charge clouds repel each other and pull the Se ions apart. The charge-density redistribution thus reveals the mechanism of $\mathrm{Se}$ atom repulsion on $\mathrm{Ru}(0001)$. Note that the shown cut of $\delta \rho(\mathbf{r})$ does not reflect the density redistribution along Se-Ru bonds because none of them is in the cutting plane. However, detailed analysis of three-dimensional isosurfaces of $\delta \rho(\mathbf{r})$ leads to the conclusion that a small amount of the electronic density is accumulated in the middle of the Se-Ru bonds providing weak covalent binding as a result of the $p S e-d R u$ hybridization discussed above.

The repulsion between $\mathrm{Se}$ atoms on $\mathrm{Ru}(0001)$ raises the question whether formation of compact Se islands is favorable in this surface. To answer this question I have calculated the energetics of detachment of a Se atom from two-, three-, and four-atom islands. The final Se configurations were obtained by separating the Se atoms from the island to the next available hcp sites on $\mathrm{Ru}(0001)$ followed by structural optimization. This makes the shortest Se-Se distance equal to $\sqrt{3} \times L(\mathrm{Ru}-\mathrm{Ru}) \approx 5.47 \AA$, where $L(\mathrm{Ru}-\mathrm{Ru})$ is the $\mathrm{Ru}-\mathrm{Ru}$ bond length in bulk Ru. An example of such a structure is shown in Fig. 5. The formation energies per atom for these structures are listed in the last row of Table I. The numbers in the table clearly show that the decomposed Se structures on $\mathrm{Ru}(0001)$ are more favorable than islands. Note that $E_{\text {form }} / n$ for $\mathrm{Se}$ in these decomposed structures is very close to the adsorption energy of a single Se atom $(5.26 \mathrm{eV})$. This reflects the fact that Se atoms do not interact noticeably with each other at such a large separation $(\sim 5.47 \AA)$. As seen from Table I, island decomposition leads to a significant decrease in $E_{\text {form }} / n(0.39 \mathrm{eV}$ for the four-atom island). A simple estimate based on this number suggests that at room temperature, the probability to meet the Se system as a decomposed structure is seven orders of magnitude higher than that for the compact Se islands.

The above estimate is valid for the system in equilibrium. To evaluate possible kinetic effects I have calculated the activation energy barriers $\Delta E_{\text {diff }}$ for a single Se atom diffusion on $\mathrm{Ru}(0001)$ and for Se atom detachment from the Se tetramer and estimated the rates of these processes within the transition state theory:

$$
R=D_{0} e^{-\Delta E_{d i f f} j k},
$$

where $D_{0}$ is the prefactor. The diffusion barriers were obtained using the drag method. ${ }^{19}$ In this approach the transition state is located by sequent displacements of the diffusing adsorbate along the assumed pathway with fixing one corresponding coordinate of the atom and letting the system relax for all other degrees of freedom. For each such a displacement the total energy and forces are calculated and analyzed and the assumed pathway is corrected. This method is very efficient for calculating the activation energy barriers for diffusion of monomer on a metal surface. For a single Se atom the calculated $\Delta E_{\text {diff }}$ is equal to $0.377 \mathrm{eV}$ for the diffusion from an hcp to fcc site and $0.266 \mathrm{eV}$ for the back fcc $\rightarrow$ hcp diffusion. Setting $D_{0}=10^{12} \mathrm{~s}^{-1}$ in Eq. (4) [which is a typical value for atoms such as Se (Ref. 20)] I obtain for room temperature $R \sim 3 \times 10^{5} \mathrm{~s}^{-1}$ and $2 \times 10^{7} \mathrm{~s}^{-1}$ for hcp $\rightarrow \mathrm{fcc}$ and $\mathrm{fcc} \rightarrow \mathrm{hcp}$ diffusions, respectively. For the Se tetramer the activation barriers are found to be equal to 0.114 $\mathrm{eV}$ and $0.504 \mathrm{eV}$ for Se atom detachment and attachment, respectively. For room temperature this makes the estimated detachment rate $\sim 10^{10} \mathrm{~s}^{-1}$ and the attachment rate nine orders of magnitude lower than that.

The above results clearly show that formation of compact Se islands on $\mathrm{Ru}(0001)$ is not favorable and the adsorbed $\mathrm{Se}$ atoms tend to separate from each other at least by a distance of $3 \times L(\mathrm{Ru}-\mathrm{Ru})$. However, such separation can be achieved for all adsorbates only if the Se coverage does not exceed $1 / 3$ ML. I should note here that energetics of these structures may be affected by long-range dispersion effects such as correlated dipole fluctuations (leading term of the van der Waals interactions) which are not taking into account by local exchange-correlation functionals used in DFT. These interactions are quite weak $(\sim 0.01 \mathrm{eV})$ (see for instance Ref. 21). They may be critical, for weak physisorption of an organic molecule on graphene or carbon nanotube. For the systems with strong ionic bonding considered here $\left(E_{b}\right.$ $\sim 5 \mathrm{eV}$ ), however, the van der Waals contribution to the binding energy is expected to be negligible.

At coverage higher than $1 / 3 \mathrm{ML}$, some Se atoms stay closer, and $E_{f o r m} / n$ decreases with the coverage. For one monolayer of $\mathrm{Se}$ on $\mathrm{Ru}(0001)$ calculations result in $E_{\text {form }} / n$ $=3.02 \mathrm{eV}$. Note that this number is larger than calculated cohesive energy of bulk Se, which is found to be $2.45 \mathrm{eV}$ suggesting that for this coverage single monolayer structure is still more preferred than bulk Se precipitations on the $\mathrm{Ru}$ surface. An attempt to calculate two Se layer structure on $\mathrm{Ru}(0001)$ resulted in decomposition of the Se layers in the course of structural relaxation. Such instability of the two layer Se structure on $\mathrm{Ru}(0001)$ may be a result of both Se-Se repulsion in the first layer and large mismatch between $\mathrm{Ru}-\mathrm{Ru}$ and Se-Se bond lengths. Similarly, Ru/Se double layer on $\mathrm{Ru}(0001)$ has been spontaneously decomposed during structural relaxation.

Deposition of other elements on Ru surfaces is also important for electrocatalysis. For example, Ru nanoparticles with Pt submonolayer coverage are reported to be efficient catalysts for hydrogen oxidation on anode of fuel cells. ${ }^{22}$ Inspired by this finding we have recently studied the geomet- 


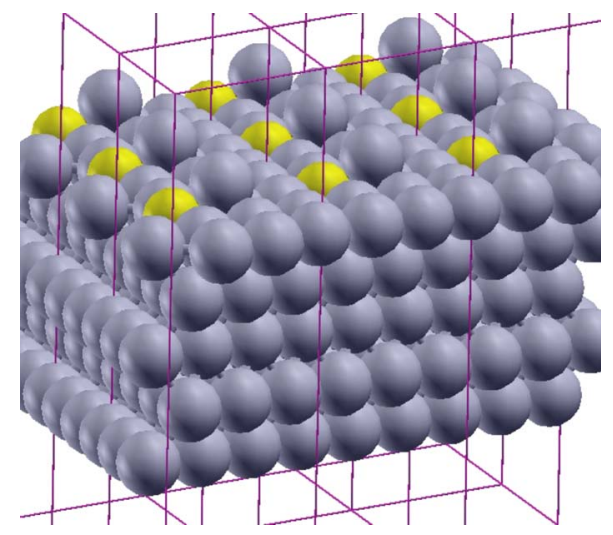

FIG. 6. (Color online) The structure obtained by exchanging Se adatom and a $\mathrm{Ru}$ surface atom.

ric and electronic structures of Pt on Ru surfaces..$^{15}$ We have found that, in contrast to $\mathrm{Se} / \mathrm{Ru}$, formation of islands is favorable for $\mathrm{Pt}$ adsorbed on $\mathrm{Ru}(0001)$. There is no significant charge transfer upon Pt adsorption on Ru. Furthermore, Pt-Pt bonds are quite strong on the Ru surface, which leads to an increase in $E_{\text {form }} / n$ with the size of the island. Such contrasting behavior is caused by a difference in the electronic states of chalcogen and transition-metal atoms. This example is to illustrate possible varieties of the structures for different elements in the submonolayer deposition regime.

The two-dimensional Se structures considered above are not the only possible arrangement of deposited atoms. In some cases, adsorbates penetrate the substrate making an alloy in the subsurface region. A possible first step of this process may be an exchange of adsorbate and surface atoms. To evaluate the energetics of this step for $\mathrm{Se}$ on $\mathrm{Ru}(0001)$, I have calculated optimized geometry of the structure with exchanged positions of adsorbed $\mathrm{Se}$ and the neighboring $\mathrm{Ru}$ atoms (see Fig. 6). The total energy of the system is found to increase by $3.15 \mathrm{eV}$ upon the Se-Ru exchange, which makes the process unfavorable. Note that this finding is in agreement with experimental evidence that $\mathrm{Se}$ is insolvable in bulk $\mathrm{Ru} .{ }^{3}$ Furthermore, the presented here calculation results suggest that alloying of Se with $\mathrm{Ru}$ is blocked at its first step that is Se-Ru exchange at the surface.

To check whether three-dimensional Se clusters can be formed on $\mathrm{Ru}$, I have calculated the energetic of 4- and $11-\mathrm{Se}$ atom pyramids on $\mathrm{Ru}(0001)$. An initial configuration of the four-atom pyramid was obtained by placing three $\mathrm{Se}$ atoms on neighboring $\mathrm{Ru}$ hcp hollow sites with the fourth atom sitting on the hollow site made up by these three $\mathrm{Se}$ atoms (see the left panel of Fig. 7). During ionic relaxation, however, the pyramid has been spontaneously decomposed. As shown in the right panel of Fig. 7, the lower layer Se atoms move away from each other to the next available fcc hollow sites while the upper $\mathrm{Se}$ atom resides on the top of $\mathrm{Ru}$ surface atom. This instability is induced by both geometrical and electronic factors. Even in initial positions, the distance of $2.733 \AA$ between the Se atoms, which form the base of the pyramid, is much larger than the bond length $(\sim 2.4 \AA)$ in bulk Se phases. The repulsion increases Se-Se separation making the hollow large enough to let the apex Se atom makes a bond with the Ru atom underneath. The latter in-
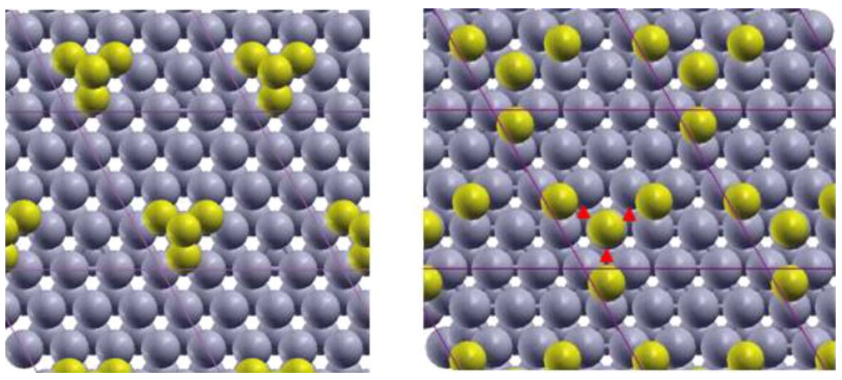

FIG. 7. (Color online) Initial configuration of the four Se atoms pyramid on $\mathrm{Ru}(0001)$ (left panel) and the decomposed structure obtained in the course of ionic relaxation (right panel). The red triangles mark the initial positions of the lower layer of Se atoms.

duces charging of the apex Se, which causes further repulsion and eventual decomposition of the pyramid.

In the initial configuration of the 11-atom Se pyramid, a seven-atom island (like those shown in Fig. 2) made up the first layer while three and one Se atoms form the second and third layers, respectively. In the course of the relaxation, this pyramid was also spontaneously decomposed. I thus find that three-dimensional Se structures, such as 4- and 11-atom pyramids are unstable on $\mathrm{Ru}(0001)$.

While all the above results have been obtained for Se atoms on the flat $\mathrm{Ru}(0001)$ surface, the $\mathrm{Se} / \mathrm{Ru}$ electrocatalysts, used for ORR, are nanoparticles which also have edges between flat facets. $\mathrm{Ru}$ atoms at these edges have lower coordination than those in the flat $\mathrm{Ru}(0001)$. One may thus still expect that Se-Se repulsion in the vicinity of the edges can be overpowered by stronger covalent bonding of Se to the low-coordinated $\mathrm{Ru}$ atoms. To test this hypothesis, I have calculated the energetic and optimized geometries for several configurations of Se adsorbed in the vicinity of the edge between the (0001) and (1101) Ru facets. The model structure of the system is illustrated in Fig. 1.

It was found that indeed $E_{b}$ of Se increases by $\sim 0.07 \mathrm{eV}$ only when it moves from the hep site in the middle of the facet to that at the edge. However, if it happens when another Se atom is placed on the (1101) facet close to the edge, the $E_{\text {form }} / n$ of the two Se structure decreases from 5.136 to $5.004 \mathrm{eV}$. If one more Se atom is placed on the (0001) facet beside the first one, as shown in Fig. $1, E_{\text {form }} / n$ decreases further to $4.904 \mathrm{eV}$. These results clearly show that Se atoms repel also in the vicinity of the edge between the Ru facets and that a small increase in Se bonding to undercoordinated $\mathrm{Ru}$ atom does not change the trend of Se adsorbates to separate from each other.

In summary, DFT-based calculations of the energetic and geometric structures have been performed for various configurations of $\mathrm{Se}$ adsorbates on $\mathrm{Ru}$ surfaces. It has been found that a significant amount of electronic charge transfers from $\mathrm{Ru}$ to Se upon selenium adsorption. As a result, Se atoms become negatively charged and repel each other. This repulsion makes compact $\mathrm{Se}$ islands on $\mathrm{Ru}(0001)$ unstable. Low detachment activation energy barriers allow Se atoms to separate easily. As the Se-Se distance increases up to $\sqrt{3}$ $\times L(\mathrm{Ru}-\mathrm{Ru}) \approx 5.47 \AA$, the repulsion between them becomes negligible. Such a separation is possible for all Se adsorbate if coverage does not exceed 1/3 ML. Further increase in Se 
coverage weakens Se-Ru bonding. Three-dimensional Se structure such as 4- and 11-atom pyramids are found to be unstable: they decompose scattering $\mathrm{Se}$ atoms over the $\mathrm{Ru}(0001)$ surface. It is also found that Se atoms tend to separate if adsorbed in the vicinity of the edge between $\mathrm{Ru}(0001)$ and $\mathrm{Ru}(1101)$ facets.

It is important to note that the obtained preferred Se structures on $\mathrm{Ru}$ may also be optimal ones for ORR. Indeed, in the intermediates of ORR, such as atomic oxygen and $\mathrm{OH}$, oxygen atoms are expected to be negatively charged, repel from $\mathrm{Se}$ adsorbates and adsorb on $\mathrm{Ru}$ sites. The $\mathrm{Ru}$ sites exposed to the surface are available at low Se coverage such as $1 / 3 \mathrm{ML}$ or less which are found to be most stable on $\mathrm{Ru}(0001)$

Therefore, Se deposition on Ru with 1/3 ML coverage or less may not only be the most stable configurations but also optimal ones for ORR because they provide $\mathrm{Ru}$ sites available for $\mathrm{O}$ and $\mathrm{OH}$ adsorption. The hypothesis, however, has to be tested and this is a subject of our ongoing studies.

\section{ACKNOWLEDGMENT}

I thank M. Alcantara Ortigoza for helpful discussions of the results of this work.
${ }^{1}$ Handbook of Fuel Cells: Fundamentals, Technology, and Applications, edited by W. Vielstich, A. Lamm, and H. A. Gasteiger (Wiley, Chichester, England/Hoboken, NJ, 2003).

${ }^{2}$ N. A. Vante and H. Tributsch, Nature (London) 323, 431 (1986).

${ }^{3}$ W. Vogel, P. Kaghazchi, T. Jacob, and N. Alonso-Vante, J. Phys. Chem. C 111, 3908 (2007).

${ }^{4}$ C. Delacôte, A. Bonakdarpour, C. M. Johnston, P. Zelenay, and A. Wieckowski, Faraday Discuss. 140, 269 (2008).

${ }^{5}$ G. Liu, H. Zhang, and J. Hu, Electrochem. Commun. 9, 2643 (2007).

${ }^{6}$ G. Zehl, G. Schmithals, A. Hoell, S. Haas, C. Hartnig, I. Dorbandt, P. Bogdanoff, and S. Fiechter, Angew. Chem., Int. Ed. 46, 7311 (2007).

${ }^{7}$ V. I. Zaikovskii, K. S. Nagabhushana, V. V. Kriventsov, K. N. Loponov, S. V. Cherepanova, R. I. Kvon, H. Bönnemann, D. I. Kochubey, and E. R. Savinova, J. Phys. Chem. B 110, 6881 (2006).

${ }^{8}$ M.-Y. Shen, S.-P. Chiao, D.-S. Tsai, D. P. Wilkinson, and J.-C. Jiang, Electrochim. Acta 54, 4297 (2009).

${ }^{9}$ A. Racz, P. Bele, C. Cremers, and U. Stimming, J. Appl. Electrochem. 37, 1455 (2007).

${ }^{10}$ K. S. Nagabhushana, E. Dinjus, H. Bönnemann, V. Zaikovskii,
C. Hartnig, G. Zehl, I. Dorbandt, S. Fiechter, and P. Bogdanoff, J. Appl. Electrochem. 37, 1515 (2007).

${ }^{11}$ A. Garsuch, X. Michaud, G. Wagner, O. Klepel, and J. R. Dahn, Electrochim. Acta 54, 1350 (2009).

${ }^{12}$ J. K. Nørskov, J. Rossmeisl, A. Logadottir, L. Lindqvist, J. R. Kitchin, T. Bligaard, and H. Jónsson, J. Phys. Chem. B 108, 17886 (2004).

${ }^{13}$ Y. Okamoto and O. Sugino, J. Phys. Chem. C 114, 4473 (2010).

${ }^{14}$ S. Stolbov, M. Alcántara Ortigoza, R. R. Adzic, and T. S. Rahman, J. Chem. Phys. 130, 124714 (2009).

${ }^{15}$ M. Alcántara Ortigoza, S. Stolbov, and T. S. Rahman, Phys. Rev. B 78, 195417 (2008).

${ }^{16}$ G. Kresse and J. Furthmüller, Comput. Mater. Sci. 6, 15 (1996).

${ }^{17}$ G. Kresse and D. Joubert, Phys. Rev. B 59, 1758 (1999).

${ }^{18}$ J. P. Perdew, K. Burke, and M. Ernzerhof, Phys. Rev. Lett. 77, 3865 (1996).

${ }^{19}$ P. J. Feibelman, Phys. Rev. Lett. 81, 168 (1998).

${ }^{20}$ G. Boisvert, L. J. Lewis, and A. Yelon, Phys. Rev. Lett. 75, 469 (1995).

${ }^{21}$ P. L. Silvestrelli, Phys. Rev. Lett. 100, 053002 (2008).

${ }^{22}$ S. R. Brankovic, J. X. Wang, and R. R. Adžic, Electrochem. Solid-State Lett. 4, A217 (2001). 